\title{
Formación en la investigación en contextos de formación militar: experiencias docentes / perspectivas de innovación ${ }^{1}$
}

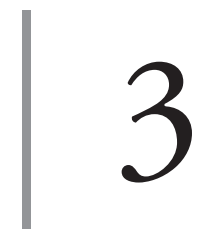

https://doi.org/10.21830/9789585241459.03

\author{
Anderson Castro Carreño ${ }^{2}$ \\ Escuela de Armas Combinadas del Ejército \\ Liliana Andrea Murillo Pardo ${ }^{3}$ \\ Escuela de Armas Combinadas del Ejército \\ Carlos Alberto Betancur Paniagua ${ }^{4}$ \\ Escuela de Armas Combinadas del Ejército
}

\section{Resumen}

El presente capítulo destaca la importancia de la investigación como pilar de la educación militar en la Escuela de Armas Combinadas del Ejército (ESACE) y describe algunos proyectos que se desarrollan en el aula con los oficiales y que fortalecen el sistema de investigación y construcción de conocimientos. El objetivo del trabajo es orientar desde su componente teórico aspectos pedagógicos y epistemológicos fundamentales desde el uso de las TIC y el pensamiento crítico, en el proceso de como se ha llevado y se ha articulado la investigación formativa y los proyectos de la ESACE. Asimismo, hace recomendaciones acordes con las necesidades de los procesos de investigación y que están dirigidas a los responsables de la formación de los oficiales,

1 Capítulo de libro que forma parte de los resultados del proyecto de investigación Práctica Pedagógica en el Ejército Nacional: Reflexiones en torno a la Formación y la Investigación, del Grupo de Investigación para la Capacitación Militar (GICAM) de la Escuela de Armas Combinadas (ESACE), registrado con el código COL0160714 de Minciencias. Los puntos de vista y los resultados de este capítulo pertenecen a los autores y no reflejan necesariamente los de las instituciones participantes.

2 Licenciado en Química de la Universidad Distrital Francisco José de Caldas, especialista en Educación y Gestión Ambiental de la misma institución y magíster en Ciencias-Química de la Universidad Nacional de Colombia. Docente de Metodología de la Investigación de la ESACE. Investigador del Grupo de Investigación en Capacitación Militar. Orcid: https://orcid.org/0000-0003-0087-8932. Contacto: andersoncastrocarreno@cedoc.edu.co

3 Ingeniera de sistemas, especialista en Investigación Universitaria de la Universidad Sergio Arboleda y magíster en Educación de la misma institución. Orcid: https://orcid.org/0000-0002-4218-3082. Contacto: lilianamurillopardo@cedoc.edu.co

4 Coronel del Ejército Nacional de Colombia. Director de la Escuela de Armas Combinadas del Ejército. Maestría en análisis de problemas políticos, económicos e internacionales contemporáneos de la Universidad Externado de Colombia. Especialización en seguridad y defensa nacionales de la Escuela Superior de Guerra "General Rafael Reyes Prieto". Profesional en ciencias militares de la Escuela Militar de Cadetes "General José María Córdova”. Orcid: https://orcid.org/0000-0002-7382-5746. Contacto: carlos.betancurpa@buzonejercito.mil.co 
en cuanto los actores directos del proceso de aprendizaje deben formarse en investigación y, más aún, las instituciones educativas deben crear un sistema de investigación que permita introducir reformas a los planes de estudio, impulsar el desarrollo de proyectos de investigación en diversas áreas del conocimiento, y participar en encuentros de ciencia y tecnología a nivel nacional e internacional.

Palabras clave: desarrollo investigativo; escuelas de formación militar; investigación; investigación formativa.

\section{Introducción}

El contexto militar es un entorno complejo caracterizado no solo por la idiosincrasia de las tareas y las funciones que se realizan, sino también por su rígida estructura jerárquica, la presencia constante de valores y normas éticas que actúan como estándares de buena conducta, así como por la preponderancia del liderazgo, la cohesión y demás elementos que destacan el perfil de un militar. Para Nuciari (2006), el ámbito militar puede considerarse un conjunto de conductas, reglas, normas y valores que se coordinan alrededor de metas (ofensivas y/o defensivas), las cuales son definidas por la sociedad en la que se desarrolla. Por su parte, Soeters, Winslow y Weibull (2006) seńalan que, más que de un contexto, se puede hablar de una cultura militar, dentro de la cual cohabitan distintas subculturas en los varios tipos de organizaciones militares que existen: armada, fuerza aérea, ejército y policía. Para estos autores, las organizaciones militares, en cuanto instituciones, representan una cultura formal aprendida, en la cual se comparten significados, ideas y símbolos específicos en los que prima la vida en común, las jerarquías, el control y la disciplina.

Para desarrollar y perfeccionar los propósitos y virtudes esbozados, se requiere además que el militar adquiera las competencias teóricas y prácticas indispensables para cumplir las funciones estipuladas para su especialidad en los cargos previstos dentro de la organización de las unidades de las Fuerza Militares. De tal manera que los programas de educación superior militar integran tanto las asignaturas en conocimientos militares básicos, exigidas para el logro de competencias necesarias específicas de las ciencias militares, como otras asignaturas complementarias provenientes de diversas disciplinas y ciencias, de acuerdo con las especializaciones del profesional requeridas para la función de seguridad, defensa y desarrollo integral de la nación.

Cabe señalar que el sistema educativo profesional militar colombiano está conformado por las escuelas de las distintas fuerzas. Estos son los centros de educación que imparten los procesos educativos de formación profesional y especializada, 
así como la constante actualización del talento humano de las Fuerzas Armadas de Colombia. Estas escuelas son:

- La Escuela Militar de Cadetes "General José María Córdova”, para el estudio de las ciencias militares que requiere la formación de la oficialidad del Ejército.

- La Escuela de Aviación Marco Fidel Suárez, donde se forman los oficiales de la Fuerza Aérea.

- La Escuela Naval Almirante Padilla, que forma a los oficiales de la Armada Nacional.

- La Escuela de Cadetes de la Policía General Francisco de Paula Santander, para la formación de la oficialidad de la Policía Nacional.

Estas Escuelas Militares y de Policía están dedicadas a formar los cuerpos de oficiales de las distintas fuerzas, quienes, por su dedicación exclusiva, brindan sus esfuerzos intelectuales y físicos para planificar, conducir, coordinar y supervisar las acciones militares propias en el campo de la defensa nacional, el orden interno y la salvaguarda de la integridad territorial.

Asimismo, la formación profesional en ciencias militares integra los desarrollos investigativos de carreras afines en otros campos del conocimiento para profundizar los conocimientos científicos, humanísticos y tecnológicos de los profesionales militares, al tiempo que se fortalece la vocación hacia la investigación en el campo de los conocimientos relacionados con la polemología.

El aporte y el liderazgo de la investigación que se realiza en el contexto militar se puede analizar desde dos corrientes conceptuales: por una parte, el enfoque de trabajo analítico, que se centra en las destrezas y habilidades requeridas para liderar individuos y organizaciones. Por otra parte, el enfoque basado en competencias, el cual estudia los planes estratégicos y metas de la organización, con el fin de identificar las competencias requeridas para implementar estrategias y alcanzar dichas metas.

En este sentido, es de suma importancia reflexionar sobre la formación en investigación e innovación que se plantea en las propuestas curriculares de la Escuela de Armas Combinadas del Ejército (ESACE) y caracterizar la formación a partir de sus planes de estudio. También es importante destacar que la buena preparación del profesorado es el indicador que más influye en la mejora de la calidad educativa en cualquier escenario académico. Para autores como Eric Hanushek (2019), la calidad de los profesores marca la diferencia en la calidad de los estudiantes, un ejemplo claro son los excelentes resultados académicos de los estudiantes en Finlandia, un caso que sirve como referencia para tomar algunos indicadores de 
calidad como muestra de un modelo de formación de los docentes orientado hacia la investigación.

\section{La investigación como parte de la formación del profesional militar en Colombia}

La formación académico-investigativa que ofrece el Ejército debe apostar por modelos educativos ligados a la práctica educativa y al desarrollo del pensamiento, de tal manera que forme líderes que tengan una visión del ámbito investigativo en pro del fortalecimiento de sus capacidades. El punto de partida de esta propuesta es el aula, la cual depende de que las facultades de educación y los centros educativos colaboren de manera necesaria y fructífera en el desarrollo de proyectos de investigación. Asimismo, es necesario que las experiencias formativas de investigación de los estudiantes se difundan masivamente por medio de congresos y encuentros, como sucede en algunas universidades de Colombia que están a la vanguardia de procesos de investigación bien estructurados y cuyos resultados son una muestra de su calidad - y por lo tanto constituyen una adecuada línea que pueden seguir algunos centros de educación militar en en el país-.

Otro aspecto importante para constituir una comunidad investigativa en el Ejército Nacional y para incrementar los procesos de investigación formativa es planear estratégicamente el uso de las tecnologías de la información y la comunicación (TIC) que favorezca su apropiación. Sin embargo, es fundamental considerar que estamos ante unos medios para la innovación, por lo cual no habría que considerar las TIC como unos fines: lo importante es la metodología, no la tecnología en sí misma.

\section{La innovación como campo en construcción}

Una de las dificultades que afronta el campo de la innovación es la falta de una teoría suficientemente desarrollada como marco de referencia para la práctica educativa innovadora y la evaluación de las innovaciones (Blanco \& Mesina, 2000). Es por esto que, al considerarse un campo de construcciones heterogéneas y dispersas en el tiempo, el término innovación se suele utilizar sin hacer explícito su significado y, por lo tanto, la utilidad y su uso en el contexto de la formación docente tienden a ser prescriptivos o carentes de rigor y precisión. El problema de su conceptualización no solo está asociado a las diferentes denominaciones inter- 
cambiables del término — cambio, mejora, reforma—, sino que además se debe a la reducción de su contenido al quedar circunscrito a la innovación tecnológica en educación y al enfoque empresarial que subordina la innovación a la mejora continua en el ámbito educativo (Barraza, 2007).

Esto último implica que cualquier ajuste a un proceso establecido se considere una acción innovadora. $\mathrm{Al}$ respecto, una de las críticas al discurso del management que asume como la innovación como un imperativo es que la convierte en un tópico rutinario aplicado a casi todo — personas innovadoras, procesos innovadores, culturas innovadoras, productos innovadores-, y que, como consecuencia, crea la identidad del emprendedor (Rodríguez, 2012; citado en Engelken, 2012).

Desde otra perspectiva, en el marco discursivo de la competitividad se enfoca la innovación hacia la incorporación de tecnologías, lo cual tiene implicaciones en las prácticas de innovación: las tecnologías se están incorporando y utilizando en las instituciones educativas desde un enfoque instrumental y técnico. No obstante, se debe señalar que contar con más tecnologías no implica necesariamente que "se estén formando consumidores críticos, o transformando sus modelos pedagógicos y prácticas culturales" (Rueda, 2008, p. 253).

En el campo específico de la formación militar colombiana, durante nuestra experiencia como docentes ha sido grato conocer los avances que el Ejército Nacional ha logrado en los últimos años en la investigación y la innovación, sobre todo en sus políticas educativas y las expectativas favorables de los planes actuales y futuros. Por ejemplo, se puede afirmar que las líneas de investigación van en una buena dirección: interdisciplinariedad, formación inicial investigadora, colaboración entre universidad y centros docentes no universitarios, apuesta por las tecnologías de la información y la comunicación, entre otros.

Se puede subrayar además la importancia que el Ejército le ha dado a la necesidad de incorporar la investigación como componente central de los programas de educación, incluyendo un componente de investigación en la formación de sus alumnos y del profesorado. Por ejemplo, el Centro de Educación Militar (CEMIL) orienta muchos de sus programas hacia la investigación, tanto así que un buen porcentaje del contenido curricular es dedicado a la formación en investigación, lo cual se evidencia en la estructura de los programas de formación inicial hasta el nivel de posgrados.

Con base en lo anterior y teniendo en cuenta visiones alternativas como la del maestro investigador en el Ejército Nacional, el práctico reflexivo y la construcción del saber pedagógico como expresión de la autonomía del docente en esta institución, se podría decir que a la investigación y la innovación se les atribuye un 
carácter transformador de la práctica docente y de las prácticas sociales inmersas en los centros de educación militar. Asimismo, con el propósito de generar y consolidar una cultura de investigación en el profesional militar en el transcurso de sus cursos académicos, resulta necesario que los centros de educación e instrucción se planteen distintas reflexiones sobre la institucionalización de las estrategias propuestas para la formación inicial: ¿`cuál ha de ser el papel de los grupos de investigación en la formación investigativa de los militares dentro del Ejército?, ¿qué implicaciones tiene la institucionalización de los semilleros de investigación y cómo se puede estimar su efecto formativo?, ¿¿deben estos ser estructuras de acogida de libre elección o un espacio curricular de los programas de formación inicial de los militares?

\section{Áreas de formación e investigación en el Ejército Nacional}

La investigación en el Ejército se ha planteado como un estudio exploratorio, en la medida en que se busca que la comunidad académica se aproxime a objetos relativamente poco explorados con el fin de que proponga nuevos objetos-problema y futuras líneas de investigación promisorias en el campo de la formación. Al respecto, cabe señalar que los estudios que identifican y analizan el impacto de los diversos itinerarios y procedimientos utilizados en la formación de futuros militares han estado creciendo.

Dentro del área de formación e investigación en el contexto militar se encuentran gran diversidad de temáticas, muchas de las cuales apuntan hacia el desarrollo de proyectos en el campo de las operaciones militares (doctrina), tales como desarrollo de armamento, aplicaciones (app, modelos virtuales, desarrollos por medio de software, etc.), mejoras a escala de procesos de ingeniería y desarrollos en temáticas de carácter ambiental, entre otros. Estas investigaciones se han formulado gracias a los procesos de asesoría que se hacen en los espacios académicos de Metodología de la Investigación. Los docentes civiles y militares orientan a los estudiantes en los procesos de formulación, desarrollo y escritura de las investigaciones, teniendo en cuenta sus inquietudes y las problemáticas provenientes de los diferentes escenarios operacionales o administrativos en los que se desenvuelven los estudiantes.

$\mathrm{Al}$ respecto cabe resaltar trabajos que han recibido reconocimientos por su calidad académica y el aporte a la fuerza. Entre ellos se pueden mencionar investigaciones sobre la mujer y su papel en el Ejército, estudios sobre algunas problemáticas como el acoso laboral y la participación en actividades que son propias del personal 
militar masculino. También existen trabajos a nivel global enfocados en estudios de estereotipos de género, de raza y resiliencia, muy importantes porque han aportado en gran medida a la construcción y mejora de los ejércitos del mundo en cuanto a sus modelos psicopedagógicos y de instrucción a partir de la integración de capacidades tanto del hombre como de la mujer en la fuerza.

En cuanto al acoso laboral, Otzvik y Rudmin (2001) exploraron las prácticas de acoso laboral en militares noruegos de diversos rangos y encontraron que hay un vacío en el conocimiento que tiene la psicología sobre al acoso laboral en ámbitos militares. Sin duda un tema de máximo interés si se tiene en cuenta que en este contexto el acoso laboral es una problemática preocupante con un altísimo riesgo, pues los protagonistas son siempre mandos superiores con falencias en el control de sus encargados.

En cuanto a estudios de estereotipos de género y raza se puede resaltar el trabajo de Biernatt et al. (1998), quienes estudiaron las creencias culturales dominantes según las cuales la habilidad de las mujeres en contextos militares es menor que la de los hombres, creencias permanecen aún en algunos escenarios. En su investigación, determinaron la influencia de estereotipos raciales y de género en los juicios y creencias de capitanes acerca de sus competencias de liderazgo y las de los otros en los entornos de la Armada de los Estados Unidos. Este tipo de trabajos permiten soportar la evidencia de estándares de desplazamiento según el modelo, en evaluaciones y juicio de competencias de liderazgo basado en el sexo y en el género. También hallaron un patrón de desplazamiento en categorías de estereotipo, en el que los hombres fueron juzgados como mejores líderes en comparación con las mujeres. A pesar de esto, se puede afirmar que estos resultados han aportado conocimiento y han permitido que los ejércitos mejoren y que la participación de las mujeres haya aumentado en el curso de las últimas décadas (Jiang et al., 2013), de manera que en los países occidentales la proporción de mujeres oficiales activas ha aumentado gradualmente y también hay una variación en los roles que desempeñan dentro del contexto militar. Precisamente, el aumento en la participación de las mujeres en el contexto militar ha generado la necesidad de desarrollar más investigaciones al respecto, ya que, como señalan Jiang et al. (2013), la gran mayoría de estudios en el contexto militar generalmente se han hecho con hombres.

Otro de los temas que se han abordado extensivamente desde la formación e investigación y que ha tomado fuerza como la psicología militar es el concepto de resiliencia, el cual integra la vivencia de las personas ante eventos estresantes, desestabilizadores y traumáticos, así como su reacción de resistencia ante estos. De acuerdo con García (2013), la definición común de resiliencia es "la capacidad del 
individuo de, no solo superar un acontecimiento traumático sino, además, salir fortalecido de él” (p. 785). Las definiciones y las expresiones de resiliencia toman especial relevancia en contextos militares, ya que se ha considerado que las labores de las fuerzas armadas conllevan más riesgos físicos y psicológicos que otros tipos de empleo. Esta situación se expresa en que los militares tienen mayor riesgo de desarrollar psicopatología como el trastorno de estrés postraumático, depresión y trastornos de ansiedad, sobre todo en aquellos soldados que participan o han participado en operaciones de despliegue militar, lo cual ha caudado tasas de bajas significativas en varios de los ejércitos del mundo (García, 2012). Todos estos estudios han permitido que los ejércitos generen programas de resiliencia basados en la evidencia y que hayan centrado sus esfuerzos en intervenir a los militares y sus familias, de tal manera que inciden de manera directa en los llamados factores internos y externos de la resiliencia: afecto y pensamiento positivo, estrategias de afrontamiento, control de comportamiento, fortaleza física, apoyo familiar, realismo, autoeficacia y cohesión grupal entre otros (García, 2013).

Otros enfoques investigativos que se deben destacar en el Ejército son aquellos que inciden en el ámbito operacional, dado que el profesional es un apoyo y toma acciones en la preparación y desarrollo de operaciones militares de combate (Staal $\&$ Stephenson, 2006). Es por esta razón que estos estudios hacen un mayor énfasis en las investigaciones y las operaciones de contraterrorismo y contrainteligencia, operaciones de inteligencia nacional enfocadas a asistir y asesorar a líderes políticos o militares para comprender las amenazas nacionales o internacionales. Asimismo, tienen un importante papel en la evaluación de amenazas internas para identificar ámbitos de alto riesgo para poblaciones o individuos que podrían constituir una amenaza interna (Williams, 2013). También se puede nombrar la investigación de Holowka et al. (2012) en contextos operativos, quienes exploraron la asociación de variables de personalidad, la exposición al combate y el presenciar o participar en atrocidades de guerra en el contexto de las intervenciones militares en Vietnam en 1.104 veteranos.

De los anteriores estudios es importante destacar sus aportes para formular nuevos interrogantes, los cuales proporcionarían campos o temas emergentes que definen objetos de investigación en diversos contextos operacionales, en los cuales, sin duda alguna, las experiencias se convierten en la mayor fuente de conocimiento. Del mismo modo, se debe señalar que las investigaciones han generado el vínculo estrecho entre autoridades de policía, las cuales han encontrado en las fuerzas mili- 
tares un valioso apoyo para enfrentar los grupos delincuenciales que han diversificado y sofisticado sus formas de operar territorialmente.

En este contexto, aunque en los últimos años Colombia ha mostrado avances en la formación de la Fuerza Pública y Militar, aún se necesitan mayores iniciativas de investigación que den respuesta a las necesidades de seguridad y defensa que requiere el país.

\section{Calidad en la educación militar: experiencias desde las aulas de la Escuela de Armas Combinadas del Ejército}

Abordar la calidad en la educación militar colombiana implica hacer un largo recorrido por nuestra historia militar y su trayectoria, cuyo transcurrir está ligado con la historia de la nación, pues las dos abrazan una sola causa, la libertad, y se proyectan hacia una sola razón, la prosperidad.

Nuestro Ejército, que nació con la patria misma, ha sido un fiel testigo y acompañante del paso a paso del devenir colombiano; ha presenciado todos los episodios que describen su memoria; ha sido un gran aportante en su desarrollo y siempre será el baluarte de lo que hoy es una República soberana, independiente y estable, fundada en el respeto por la dignidad humana, bajo los principios de una democracia participativa y el imperio del estado de derecho.

Es así como este trabajo pretende demostrar que, a través de su historia, el Ejército Nacional ha sido un pilar de la estabilidad democrática en Colombia y que en esta misión los procesos educativos establecidos en la institución han tenido un papel protagónico, ya que han brindado una educación militar eficaz que siempre ha tenido como pilar de la formación los principios y valores. A pesar de las equivocaciones en las que han incurrido miembros de la institución, se puede decir que el Ejército ha sabido enfrentar las crisis de diverso orden y ha sido un factor de estabilización.

La institución militar ha sido un actor fundamental en el mantenimiento de la democracia, pues a pesar de las diversas amenazas se ha sostenido aun por encima de potenciales enemigos que han querido destruirla. Es por esto que merece el reconocimiento de la sociedad colombiana, dado que la institución militar ha contribuido de gran forma a la construcción y consolidación de la República.

Teniendo en cuenta lo anterior, para el Ejército Nacional mantener la legitimidad implica entender y asumir una serie de compromisos y valores que incondicionalmente proveen el alimento de su subsistencia, pero sin descuidar el uso de 
la fuerza, ya que su imperio resulta virtuoso, tal como lo afirma nuestro Libertador Simón Bolívar: "Mirad que sin fuerza no hay virtud y sin virtud perece la República".

Precisamente en este sentido la formación y la educación militar cumplen un papel fundamental: gran parte de la victoria o la derrota de un ejército radica en la competitividad de sus mandos, pues son ellos quienes deben conducir a la tropa que obedece en estricta disciplina. Por ello, más allá del entrenamiento militar, que tiene como característica la adquisición de una destreza mecánica para ejecutar una maniobra, está la formación, cuyo fundamento radica en aprender comportamientos que redunden en beneficio de la legitimidad.

Ahora bien, para analizar la educación militar en el Ejército Nacional desde la experiencia de las aulas de la Escuela de Armas Combinadas, se deben tener en cuenta aspectos trascendentales en la evolución de los procesos formativos sucedidos a través de la historia de la educación en los ámbitos militares. El eje del estudio de la formación y educación militar es la evolución que ha tenido la Escuela de Armas Combinadas del Ejército, ya que las constantes innovaciones en su programa curricular han contribuido a mejorar la calidad profesional de sus estudiantes, lo cual ha permitido que se mantengan las riendas de un Ejército victorioso, sujeto a la autoridad civil, respetuoso de la Constitución y la ley, y enmarcado en los principios democráticos de la nación. De este modo se puede evidenciar cada uno de los sucesos que hacen posible que los oficiales y suboficiales alumnos de esta escuela de formación, educados en una sucesión de generaciones, sean los encargados de perpetuar la heredad de una sublime empresa, que con desvelo y sin nada a cambio trabaja para alcanzar una democracia permanente para los colombianos.

Como se denota, se ha tenido en cuenta la trascendencia de los cambios históricos institucionales que ha tenido el Ejército Nacional, sus principales reformas, doctrinales y académicas, en las cuales ha recibido el apoyo de algunas misiones extranjeras que contribuyeron con la organización y formación de la doctrina. Estas importantes restructuraciones permitieron que los procesos formativos evolucionaran, de manera que son hitos históricos de la formación militar y del desarrollo del Ejército, pues le han permitido contribuir a la nación por medio de la formación democrática de sus oficiales y suboficiales, los principios y valores institucionales, la transversalidad entre el estudio de las ciencias militares y los procesos educativos del Estado, hasta llegar a la actualidad, donde la educación militar ha alcanzado altos estándares de calidad que la ha llevado a destacarse en el medio latinoamericano.

La Escuela de Armas Combinadas del Ejército es por tradición la institución de educación, formación y capacitación de los oficiales de grado capitán y teniente, 
así como de los suboficiales que van desde el grado de cabo primero hasta el grado sargento mayor, quienes reciben su formación académica y militar, como también su capacitación para su respectivo ascenso al grado inmediatamente superior. Esta formación se enmarca en "los estudios que se adelantan, dentro del marco de la formación militar por competencias en la disciplina de las ciencias militares, así como la capacitación técnico-científica y especialista, se encuadran en el rango de la educación superior de la nación" (Ejército Nacional, 2016) conforme a la reglamentación que se disponga para el efecto. La Escuela cuenta con un Proyecto Educativo Institucional (PEI) de suprema claridad que parte de la propia naturaleza de la Escuela, su contexto histórico y el de la nación. Además, las líneas académicas que orientan y encauzan con precisión el desarrollo educativo se trazan con base en los principios y valores democráticos en aras de obtener un selecto grupo de profesionales capacitados con una excelente formación por competencias al servicio de la nación y sus ciudadanos.

Siendo así, es importante considerar que los lineamientos institucionales inciden positivamente en la optimización de los procesos administrativos de la Escuela de Armas Combinadas, y por ende en la sociedad, ya que el fin último de todo programa educativo es formar personas íntegras, no solo en el ámbito profesional, sino también en los componentes ético y moral. Es decir, se busca que sean individuos comprometidos con la transformación de la comunidad en pro del bien común, sobre todo considerando que se trata de una organización pública, ya que su razón de ser está relacionada con el progreso social, económico y cultural de una sociedad. Al respecto cabe mencionar el interesante planteamiento formulado por Pedroza y Massé (2009), quienes consideran que la sociedad tiene un orden, pero que es vulnerable, sujeto a distintas variables con sus propias características, que surgen entre las interacciones del orden y desorden, del determinismo y el azar.

La estructura curricular de cualquier área de estudio es su columna vertebral, que integra los saberes, contenidos y procedimientos del programa. Por tal razón, una débil organización o modificación incide directamente en la formación integral del estudiante, así como en la consecución de los objetivos de estudio planteados y, a la postre, en el desempeńo laboral. En parte, esto se debe a que la concepción antigua del currículo lo entendía solamente como la suma del contenido temático, los créditos y la malla curricular, cuando en realidad debe englobar también el contexto educativo en que se desenvuelve el estudiante, el marco socio-cultural y económico, y en este caso específico el ámbito militar.

Particularmente, en el contexto colombiano, la Escuela de Armas Combinadas está llamada a cumplir un importante papel en el aumento de la capacidad inno- 
vativa de la institución, mediante el desarrollo de conocimientos y habilidades en sus estudiantes, resultado del proceso de enseñanza-aprendizaje y a través de la generación de productos y procesos capaces de dar respuesta a las necesidades del Ejército. En concordancia con lo anterior, la Escuela de Armas Combinadas del Ejército desarrolla un intenso y sostenido proceso de gestión con el fin de motivar, captar, generar y darle visibilidad a las innovaciones que hacen los estudiantes, un equipo multidisciplinario de profesores y estudiantes, con el apoyo de personal administrativo y obrero. Este personal ha llevado a cabo diversas actividades con logros importantes en el desarrollo del potencial innovador, lo cual se evidencia en un contingente de estudiantes que han producido distintos proyectos innovadores que dan respuesta a problemas y necesidades institucionales.

Estos resultados constituyen un aporte que profundiza en la formación de la cultura de la innovación, al concebirla como una actividad que involucra — junto a la formación de talentos, las capacidades y los recursos disponibles — una manera particular de ver el mundo, sus representaciones, la identidad, las expectativas y los modos de trabajo. Por esta razón la educación de nuestra escuela debe dirigir esfuerzos deliberados y sistemáticos para que los estudiantes se formen dentro de una concepción de participación y protagonismo innovador en los contextos en los cuales deban desempeñarse como oficiales del Ejército Nacional.

\section{Metodología}

En este orden de ideas, los investigadores consideran que una perspectiva cualitativa es la más adecuada puesto que reconoce el valor del conocimiento como una construcción en la que media la experiencia de los sujetos que dan cuanta del mundo en el que vive. En este sentido, el acercamiento a la realidad se hace desde los preceptos de la hermenéutica, que ofrece a los investigadores profundidad de significados:

- Amplitud del enfoque.

- Riqueza interpretativa.

- Contextualiza el fenómeno.

- Puede desarrollar preguntas e hipótesis antes, durante y después del proceso de recolección y análisis de datos.

- Los datos recolectados poseen credibilidad, confirmación y valoración.

- Permite descubrir, construir e interpretar una realidad (Álvarez, 2011). 
Este proceso metodológico permite integrar las teorías y los ejes temáticos afines al saber específico del aprendizaje. Asimismo, esta perspectiva metodológica permite tener en cuenta la diversidad de opiniones y facilita el diseńo de la investigación, al combinar la información teórica con la obtenida en campo. De esta manera es más sencillo interpretar y analizar los datos, al tiempo que se logra complementariedad en la información —es decir, que se corroborare mutuamente—, lo cual permite formular conclusiones veraces. Además, garantiza un alcance macro, ya que el resultado permite realizar propuestas que aporten grandes soluciones a la problemática (Salazar, 2011).

\section{Experiencias investigativas en las aulas de la Escuela de Armas Combinadas del Ejército}

Como ha sido demostrado en el transcurso de los años, la Escuela de Armas Combinadas ha sido el centro de formación por el que pasan todos los oficiales y suboficiales del Ejército, en donde adquieren conocimientos relacionados con las competencias que necesitan para alcanzar el grado inmediatamente superior.

Es así como la Dirección de la Escuela y, de manera particular, el Departamento de Ciencia y Tecnología ha evaluado y sistematizado las experiencias de formación en cada uno de los cursos de ascenso y especializaciones. Los datos obtenidos de las evaluaciones de carácter cualitativo y cuantitativo han mostrado una mayor disposición e interés por parte de los estudiantes para abordar diferentes problemas.

A continuación se exponen brevemente dos trabajos de investigación que reúnen elementos de innovación y que pueden aportar a la fuerza.

\section{Estudio de optimización de la movilidad y trasporte de la aviación ejército}

Este trabajo fue realizado en el Curso Comando de Aviación en el año 2009 por los estudiantes Yeinson Arley González Correa, Julián Andrés Quintero Pérez, Diego Bonilla Arias y Diego Peńa Mosquera.

El proyecto está basado en un análisis estadístico sobre las experiencias personales en misiones de aviación y la normatividad para el transporte de personal y material en la aviación del Ejército. La investigación identificó las características de las misiones que no optimizan el uso de las aeronaves de la Aviación del Ejército y con base en estos resultados se hizo una propuesta simple y asequible de una app que puede identificar, coordinar, regular y establecer movimientos de pasajeros y 
carga disponibles en futuros vuelos de la Aviación del Ejército, de manera que la misión principal o razón del vuelo permita llevar personal y material adicional sin afectar la misión. Esta iniciativa permite que los miembros de las FF. MM., las familias y los civiles al servicio del Ministerio de Defensa puedan ser transportados sin costo alguno, lo cual ayuda a la institución a ser más eficiente en la movilidad y el trasporte de personal y carga operacional, como también a elevar el bienestar de personal que necesite el apoyo aéreo.

La investigación concluye que a partir de los problemas existentes en la fuerza, como la falta de coordinación, conocimiento y coordinación entre los responsables de planear y ejecutar vuelos en la aviación del Ejército y los demás miembros de la institución, las aeronaves deben ser utilizadas en pro de un mejor aprovechamiento, sea operacional o administrativo, como los movimientos de personal por término de vacaciones. El caso es que con la voluntad y la tecnología que nos permita direccionar este proceso podríamos incluso llegarlo a implementar en todas las aeronaves del Estado colombiano, de manera que se mitigarían riesgos de personal y bajarían gastos de funcionamiento y pasajes para personal militar y civil (figura 1).

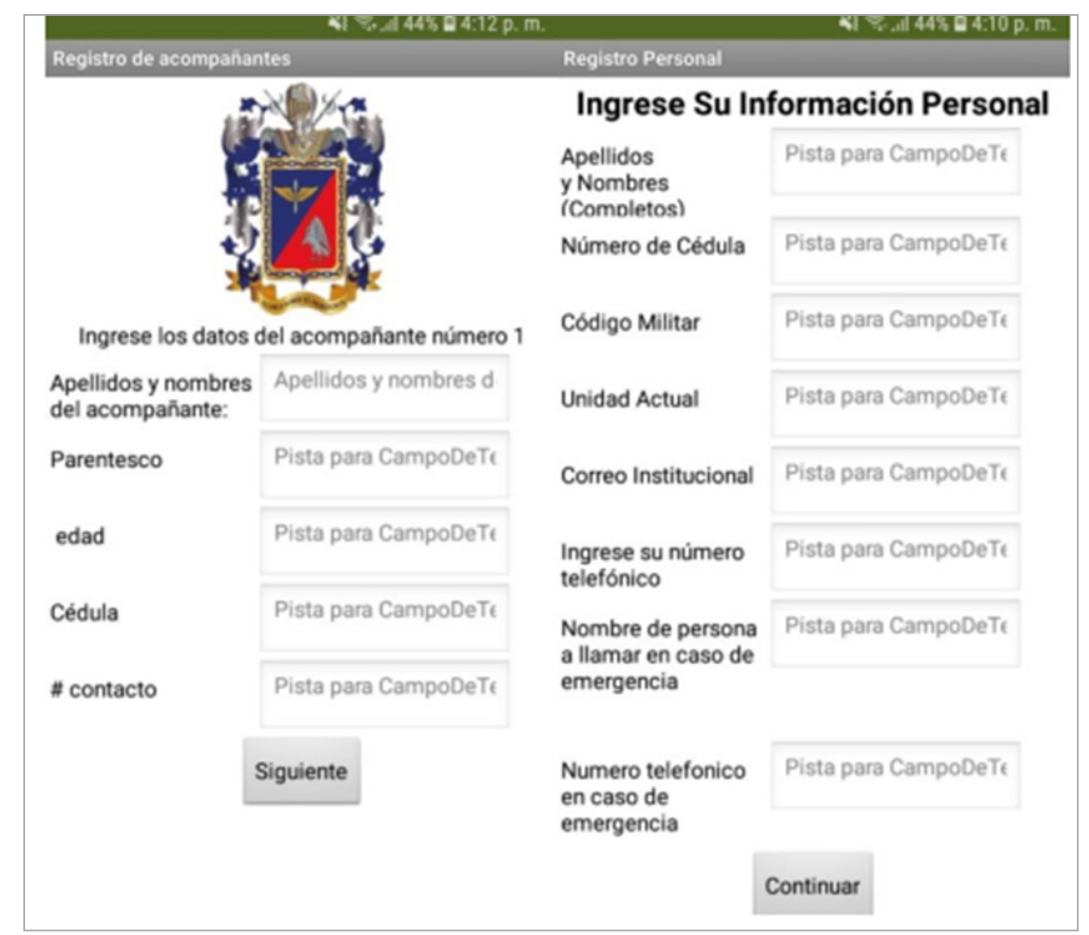

Figura 1. Producto de la app desarrollada.

Fuente: Original de los autores. 


\section{Implementación de tecnología en nano tubos de carbono en guantes de protección, aplicados para el desminado}

Este proyecto (figura 2) fue realizado en el Comando C2019 por el CT. Carlos Franco Ocampo, el CT. Jeisson Gerenas Díaz, el CT. Marlon González Rodríguez y el CT. Oscar Gutiérrez Rodríguez.

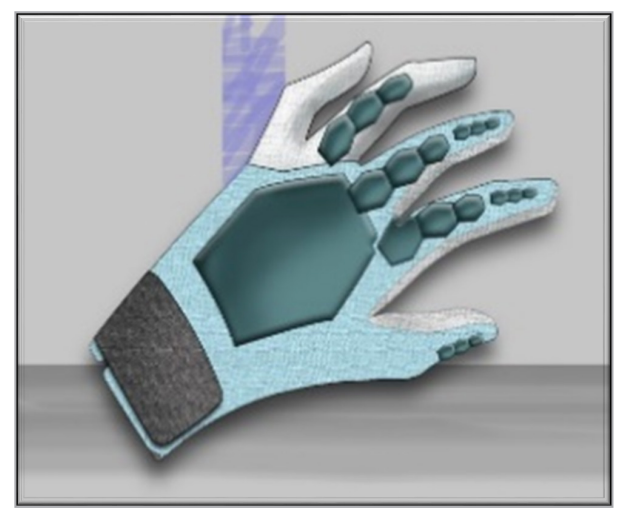

Figura 2. Diseño del guante con nano tubos.

Fuente: Original de los autores.

Según los últimos datos presentados por el programa Descontamina Colombia ${ }^{5}$ (Oficina del Alto Comisionado para la Paz), la mayoría de las víctimas de artefactos explosivos en los últimos años de conflicto armado en Colombia han sido los miembros de la Fuerza Pública y prevalece la afectación a los campesinos

Es así como este problema se ha convertido en uno de los más relevantes del país en cuanto a orden público. Anualmente, los artefactos explosivos matan o hieren a más de mil colombianos. Por ello, el Gobierno Nacional, en conjunto con la comunidad internacional, ha querido acabar con este tipo de armas aprobando leyes, procedimientos y procesos enmarcados en normas internacionales aplicables a la problemática de las minas.

El diseño metodológico de la investigación fue experimental, toda vez que se comprobó por medio de la experimentación la eficiencia de los componentes, las texturas y se validó las mezclas químicas que permitieran mejorar la resistencia de las fibras.

En cuanto a los antecedentes, el proyecto señala que el Ministerio de Defensa Nacional, con la creación de los Batallones de Desminado Humanitario, pretende

5 El último informe presentado por el programa Descontamina Colombia es del 31 de diciembre de 2019. http://www.accioncontraminas.gov.co/estadisticas/Paginas/victimas-minas-antipersonal.aspx 
mitigar los efectos de los Artefactos Explosivos Improvisados (AEI) y las minas antipersonales con unidades de desminado destinadas a realizar procedimientos técnicos minuciosos que permiten su destrucción en los diferentes departamentos a lo largo y ancho del territorio nacional. Dicho proceso de desminado se realiza con tres técnicas: manual, canino y mecánico, y actualmente el desminador cuenta con elementos de protección en todo el cuerpo, excepto los brazos y manos. Esto hace reducir su eficiencia en el procedimiento, ya que está expuesto a cualquier tipo de lesiones que puedan ocasionarle la manipulación del terreno y/o los implementos necesarios para realizar el procedimiento.

Por este motivo, el objetivo del trabajo fue diseñar un prototipo de guante de protección personal que ofrezca mayor seguridad en los procedimientos de desminado manual en las unidades de Desminado Humanitario en Colombia. Específicamente, se pretende implementar esta tecnología en un guante con características técnicas para que proteja las manos hasta cierto grado de explosión de artefactos explosivos de bajo poder. De tal forma que con el uso de estos guantes de protección se pueda mitigar el riesgo de sufrir daños o afectaciones en cuanto a quemaduras y desmembramientos de las falanges. De esta forma, sería posible incrementar la efectividad en los procedimientos de desminado manual (figuras 3 a 7).
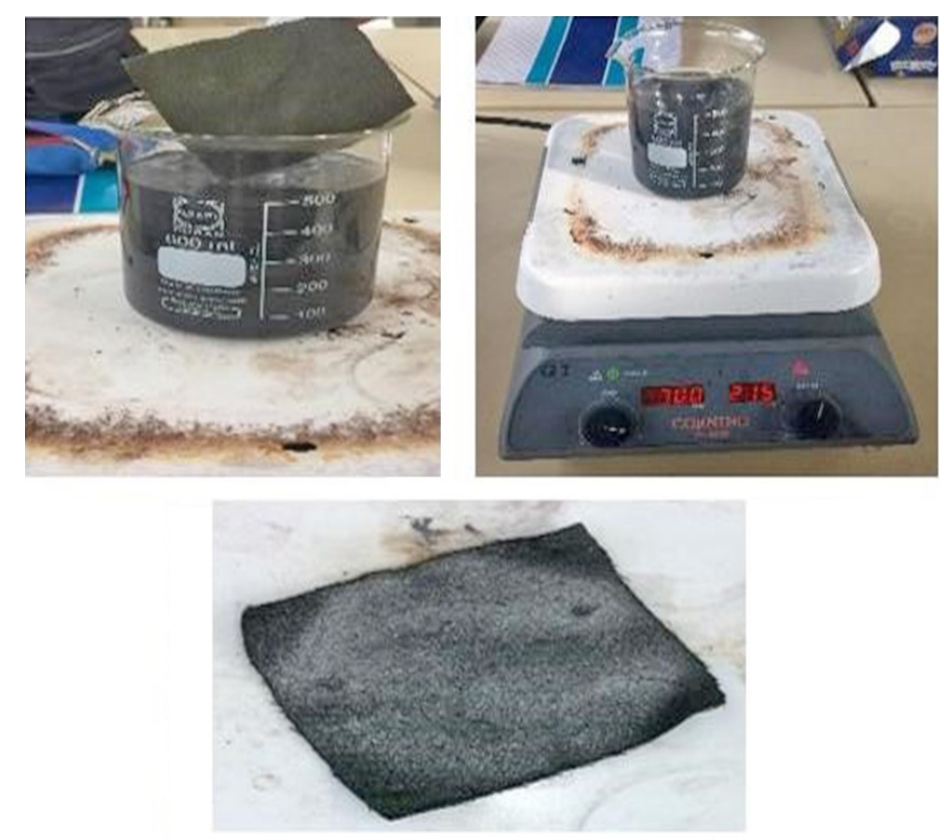

Figura 3. Proceso de sintetización.

Fuente: Original de los autores 

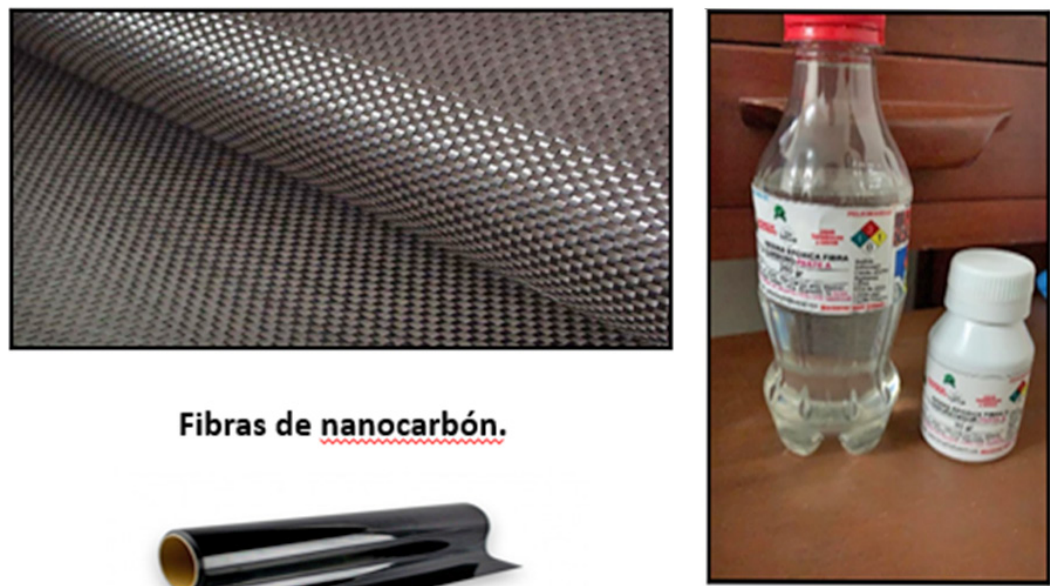

Fibras de nanocarbón.

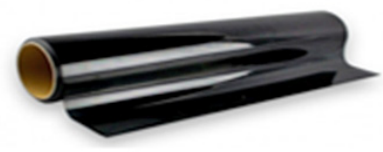

Resina de carbono

Figura 4. Materiales.

Fuente: Original de los autores.

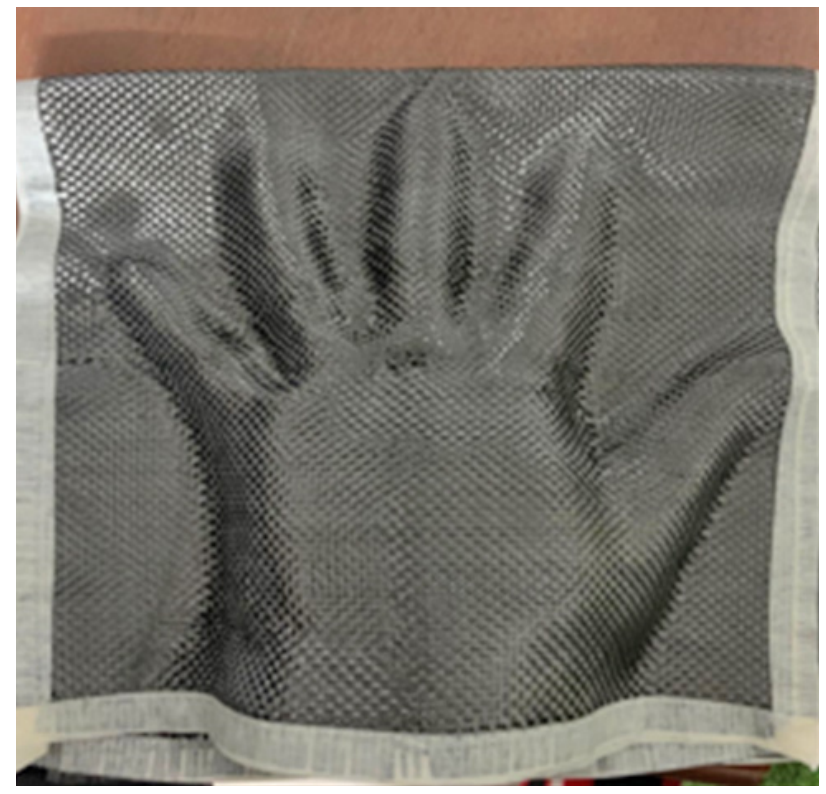

Figura 5. Molde en fibra de carbono recubierto, resistente al calor para proteger las manos. Es esencial para evitar algún daño en la piel.

Fuente: Original de los autores. 

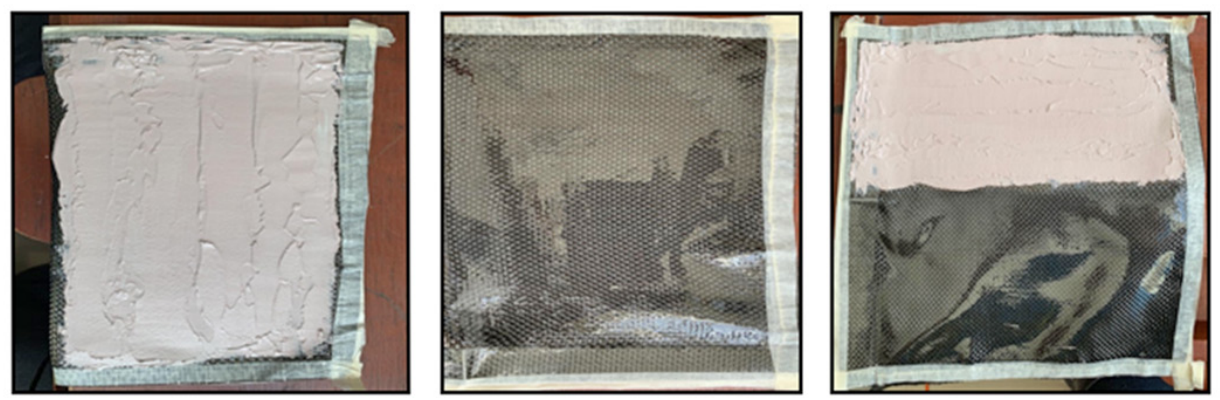

Figura 6a. Exposición del material.

Fuente: Original de los autores.

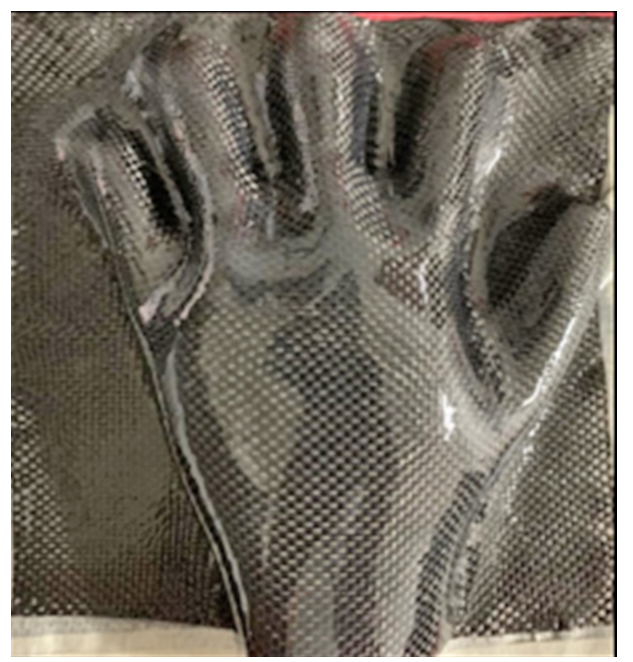

Figura 6b. Exposición del material.

Fuente: Original de los autores.
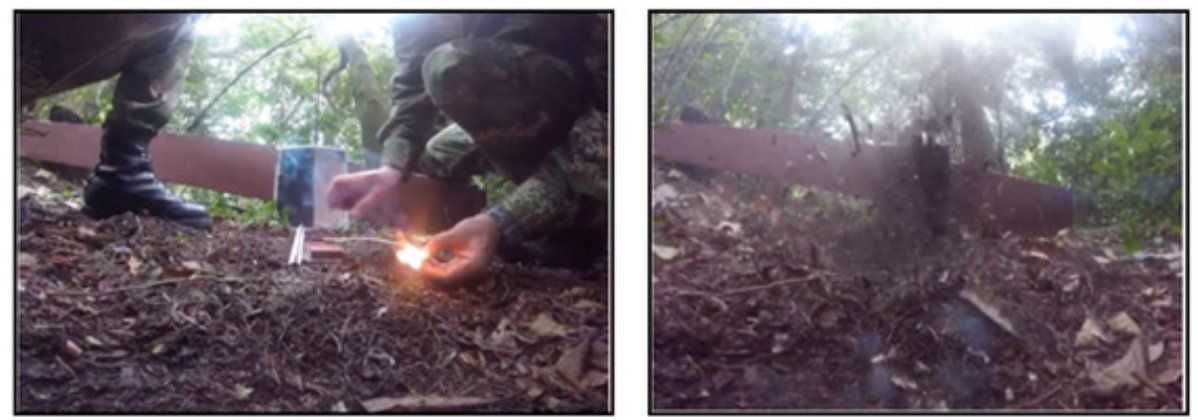

Figura 7. Reacción ante un detonador.

Fuente: Original de los autores. 


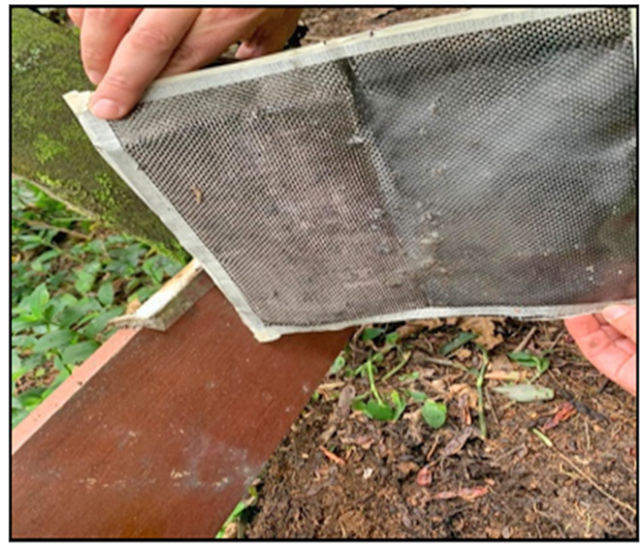

Figura 8. Resultado final después de las explosiones de temperatura y alzas de calor. Fuente: Original de los autores.

\section{Conclusiones}

De acuerdo con la investigación, las partículas de nano carbono adheridas a la tela con la que se elaboró el guante para desminado aumentan la protección al fogaje de hasta $75^{\circ} \mathrm{C}$ que emana la explosión. Esto evita que la piel se afecte con quemaduras de hasta tercer grado y protege a la persona de posibles ataques de animales ponzońosos, plantas con espinas, líquidos venenosos, desechos metálicos y esquirlas de los restos explosivos de guerra.

Teniendo en cuenta las teorías relacionadas con las explosiones de los artefactos explosivos, los trajes antiminas que existen no protegen de desmembramiento de ninguna parte del cuerpo, pero sí de afectaciones directas relacionado con el riesgo de quemaduras y reducción de impacto directo a la piel de las esquirlas o metralla.

\section{Agradecimientos}

Esta investigación ha sido realizada gracias al apoyo de la Escuela de Armas Combinadas del Ejército (ESACE). Los autores declaran que no existe ningún potencial conflicto de interés relacionado con este capítulo.

\section{Financiación}

Los autores no declaran fuente de financiación para la realización de esta investigación. 


\section{Referencias}

Álvarez, C. A. (2011). Metodología de la investigación cuantitativa-cualitativa, guía didáctica. En M. Á. Arturo. Universidad Sur Colombiana, Neiva, p. 10.

Barraza Macías, A. (2007). Una conceptualización comprehensiva de la innovación educativa. Innovación educativa, 5(28), 19-31.

Blanco, R. \& Mesina, G. (2000). Estado del arte sobre las innovaciones educativas en América Latina. Santafé de Bogotá: Convenio Andrés Bello.

Biernatt, M., Crandall, C., Young, L., Kobrynowicz, D. \& Halpin, S. (1998). All that you can be: Stereotyping of self and others in a military context. Journal of Personality and Social Psychology, 75, 301-317.

Ejército Nacional. (2016). Proyecto Educativo Institucional. Bogotá, D. C.: Ejército Nacional.

Engelken, M. (2012) Introducción. En L. Merino Malillos (coord.), Contextos y uso de la innovación social (pp. 115-122). Bilbao: Servicio Editorial de la Universidad del País Vasco. Recuperado de http://www.academia.edu/1895786/

García Silgo, A. (2013). Revisión de programas de resiliencia basados en la evidencia en los ejércitos. Sanidad Militar, (69), 182-194.

Hanushek, E. (2019). The value of smarter teachers: international evidence on teacher cognitive skills and student performance. J. Human Resources (54), 857-899. doi:10.3368/ jhr.54.4.0317.8619R1

Holowka, D., Wolf, E., Marx, B., Foley, K., Kaloupek, D. \& Keane, T. (2012). Associations among personality, combat exposure and wartime atrocities. Psychology of Violence, (2), 260-272.

Jiang, Y., Sun, Y., Yang, Y., Tang, J., Wu, S., Miao, D. \& Zhu, X. (2013). Gender differences in coping styles of chinese military officers undergoing intensive training. Military Psychology, (25), 124-135.

Nuciari, M. (2006). Models and explanations for military organization: an updated econsideration. En G. Caforio. (ed.), Handbook of the sociology of the military (pp. 61-87). Nueva York: Springer.

Otzvik, K. \& Rudmin, F. (2001). Bullying and hazing among Norwegian army soldiers: two studies of prevalence, context, and cognition. Military Psychology, (13), 17-39.

Pedroza Flores, R. \& Massé Narváez, C. (2009). Educación y universidad desde la complejidad en la globalización. México: Casa del Libro.

Rodríguez Jiménez, Y. J. (2009). La formación de docentes investigadores: lineamientos pedagógicos para su inserción en el currículo. Revista Teoría y Praxis Investigativa, 4 (1), 25-32.

Rueda, R. (2008). Cibercultura/es: capitalisme cognitiu i cultura. Temps d' Educació, 34, 251-264.

Salazar, G. L. (2011). La metodología de la investigación y sus enfoques. En G. S. Latorre. Cale: Norma.

Soeters, J., Winslow, D. \& Weibull, A. (2006). Military culture. En G. Caforio. (ed.), Handbook of the sociology of the military (pp. 237-255). Nueva York: Springer.

Staal, M. \& Stephenson, J. (2006). Operational Psychology: An emerging subdiscipline. Military Psychology, (18), 269-282.

Williams, T. (2013). Operational psychology. En B. Moore \& T. Barnett (ed), Military psychologists desk reference (pp. 62-66). Nueva York: Oxford University Press. 